\title{
A valoração do Estágio Supervisionado na Unidade de Saúde da Família pelos alunos de Odontologia: quais fatores influenciam sua percepção?
}

\author{
Pedro Augusto Thiene Leme*; Marcelo de Castro Meneghim**; Antonio Carlos Pereira**; Karine \\ Laura Cortellazzi**; Glaucia Maria Bovi Ambrosano**; Fábio Luiz Mialhe** \\ * Doutor em Odontologia, Universidade Estadual de Campinas \\ * Departamento de Odontologia Social, Faculdade de \\ Odontologia de Piracicaba da Universidade Estadual de \\ Campinas.
}

Recebido em 24/07/2017. Aprovado em 03/10/2017.

\begin{abstract}
RESUMO
O estágio extramuros é uma importante estratégia pedagógica utilizada na formação das profissões da saúde no sentido de fomentar uma prática mais integral e compreensiva do ponto de vista social, sobretudo quando desenvolvido em cenários reais de serviços públicos do Sistema Único de Saúde. Essas experiências nem sempre são bem aceitas pelos alunos, de forma que explorar os fatores que interferem nessa valoração pode ser útil para aprimorar essa atividade. O objetivo do presente estudo foi explorar quais percepções dos alunos de um curso de Odontologia influenciam a valoração a respeito da importância do estágio em Unidades de Saúde da Família na sua formação profissional. Um total de 185 alunos que realizaram estágio durante seus últimos anos de graduação participaram da pesquisa. Os dados foram obtidos por meio de formulários eletrônicos compostos por questões sobre o funcionamento e condições de trabalho nas unidades de saúde (variáveis independentes) e pela valoração de importância atribuída ao estágio (variável dependente). Os alunos que perceberam falta de material de consumo $(\mathrm{OR}=2,17, \mathrm{IC}=1,20$ - 4,00), limpeza e higienização incompatíveis com a prestação de serviços de saúde $(\mathrm{OR}=7,69,=2,32-25,00)$ e a falta de respostas satisfatórias por parte dos trabalhadores das equipes de saúde aos usuários $(\mathrm{OR}=4,34, \mathrm{IC}=1,63-11,11)$ tiveram maiores chances de considerar a experiência como não-importante. Concluiu-se que problemas infraestruturais e aporias do cotidiano dos serviços parecem influenciar numa valoração negativa do estágio por parte dos alunos, apontando a necessidade de um maior apoio do corpo docente para transformar essa leitura da realidade em produtos pedagógicos importantes aos alunos.

Descritores: Educação em Odontologia. Desenvolvimento de Pessoal. Sistema Único de Saúde. Saúde da Família.
\end{abstract}

\section{INTRODUÇÃO}

Desde meados da década de 1990 vem sendo observada no Brasil a expansão da atenção básica, reconfigurada por meio da Estratégia de
Saúde da Família (ESF) que, em oposição aos modelos tradicionais de organização do cuidado em saúde, buscou aprimorar uma organização em rede que melhor respondesse às demandas da 
população ${ }^{1,2}$. Essa reorganização demanda algumas competências que nem sempre são desenvolvidas nos ciclos de formação dos profissionais de saúde, normalmente calcados em práticas liberais e privativistas, em detrimento a uma atuação de promoção de saúde. Assim, é demandada uma visão ampliada sobre os determinantes sociais, uma compreensão sistêmica e integral em níveis individuais e coletivos, incluindo a família, o que requer também uma formação profissional adequada para tal ${ }^{3}$.

No Brasil, a discussão sobre a educação profissional para a saúde tem sido tema recorrente nas esferas da formação e dos serviços nas últimas décadas, motivado por um intenso debate no campo sanitário sobre a necessidade de alteração do cenário em que os profissionais de saúde são formados. Segundo a perspectiva da Saúde Coletiva, estes cenários historicamente privilegiam o paradigma biomédico, com ausência de debate relativo ao cuidado ampliado em saúde, com desligamento da realidade social e epidemiológica, em modelos curriculares fragmentados, dependentes da alta tecnologia, limitados pelo enfoque pedagógico baseado em metodologias tradicionais de transmissão de conhecimentos, pela inserção tardia do estudante no mundo do trabalho, dentre outras limitações ${ }^{4}$.

Desta forma, as ações no sentido de promover mudanças curriculares nos cursos das áreas da saúde que respondam a essa necessidade, vêm surgindo e uma das principais estratégias é a utilização dos estágios curriculares supervisionados, sobretudo o estágio extramuros realizado nos ambientes de trabalho dos serviços que compõem o Sistema Único de Saúde (SUS) ${ }^{4}$. Para além do país, existem também experiências pedagógicas de inserção de graduandos em Odontologia nos serviços locais de saúde, fazendo-os vivenciar a realidade. Tais experiências, mesmo que não representem uma "revolução curricular", uma vez que sobrevivem, via de regra, isoladas frente às disciplinas hegemônicas e convivem com o fenômeno do currículo oculto, vêm mostrando resultados positivos $^{5-9}$. Aposta-se no potencial dos estágios extramuros per se de estimular o senso crítico, o desvelamento da realidade social, apontar oportunidades positivas de trabalho e despertar o interesse dos alunos para atuar no setor público, esperando-se que contribua para a formação de um profissional com maiores possibilidades de ampliar sua clínica, sendo mais resolutivo, mais efetivo e competente do ponto de vista epidemiológico e social.

É um desafio efetivar propostas pedagógicas contra hegemônicas, num contexto acadêmico que, muitas vezes, desautoriza experiências alternativas à rigidez flexneriana, cientificista, colocando em constante tensão sua sustentabilidade $^{10}$. Assim, torna-se útil explorar os estágios extramuros que puderam se concretizar como objeto de análise, extraindo elementos que aperfeiçoem as experiências futuras.

Para este estudo, partimos da hipótese de que, para os alunos, a percepção negativa sobre a infraestrutura (no caso, de Unidades de Saúde da Família - USF), exerce interferência na valoração que atribuem à experiência como um todo, a despeito do objetivo do estágio ser, justamente, o contato com a realidade, ainda que imperfeita, dos serviços. Uma melhor compreensão sobre esse tema poderá ser utilizada pelas disciplinas para intervir no ensino da Odontologia em Saúde Coletiva, possivelmente trabalhando sobre as expectativas artificiais geradas no interior da academia, de forma que os alunos consigam compreender a experiência sob uma perspectiva menos técnica e mais política e social, elementos fundamentais para embasar uma ampliação da clínica.

Assim, o objetivo do presente estudo foi 
explorar quais percepções influenciam a valoração do aluno a respeito da importância do estágio na sua formação profissional.

\section{METODOLOGIA}

Estudo descritivo, do tipo observacional transversal. Os sujeitos da pesquisa foram todos os 185 alunos do quarto ano de graduação de um curso de Odontologia de uma faculdade pública do estado de São Paulo, provenientes de três turmas, as quais participaram de atividade extramuros obrigatória em USF no período compreendido entre 2008 e 2010. Os alunos permaneceram 64 horas nas USF, sendo uma semana (32h) em cada semestre. Como atividades pedagógicas, observaram e participaram de grupos de discussão sobre saúde bucal, visitas domiciliares com os agentes comunitários de saúde, reuniões semanais de equipe, atendimento clínico, programas escolares, territorialização e levantamentos epidemiológicos. Essas atividades foram programadas com o objetivo de facilitar, ao aluno, avaliar e adotar uma abordagem integral do processo saúde-doença, planejar as ações preventivas e curativas para a população cadastrada e oferecer a oportunidade de conhecer e desenvolver experiências e estudos no contexto dos serviços de saúde com ênfase em clínica ampliada $^{2,11,12}$.

Os alunos deveriam responder, ao final dos ciclos de estágio de cada semestre, a um formulário de avaliação, disponível em ambiente virtual de aprendizagem. Este formulário foi concebido pelos docentes responsáveis pela disciplina para ser um mecanismo de feedback e promover a reflexão dos estudantes sobre a experiência vivenciada, sendo parte de um conjunto de instrumentos utilizados para avaliar a experiência, que, para além do escopo deste estudo, considerou a perspectiva de diferentes atores (estudantes, tutores e trabalhadores do serviço $)^{13}$. Este formulário foi composto por questões fechadas sobre o funcionamento $\mathrm{e}$ condições de infraestrutura, utilizadas como variáveis independentes. No final do instrumento constava a questão "Qual a sua opinião pessoal em relação à importância desse estágio na USF para a sua formação profissional?", que possuía um campo para resposta fechada ("muito importante", "importante", "razoável", "indiferente" e "muito indiferente"), fornecendo a variável dependente "importância atribuída" para o presente estudo.

Para a análise, foram agregados os dados de todos os formulários disponíveis, totalizando 360 registros de 185 estudantes. Como este formulário era eletrônico, provavelmente por erro ou dificuldade de preenchimento, alguns alunos deixaram algumas respostas em branco, as quais foram consideradas como dados ausentes durante as análises estatísticas. Por este motivo, nos resultados, a soma do número de respostas para algumas variáveis não atinge este número. Além disso, a pergunta "A estrutura física é adequada?" não foi aplicada no primeiro semestre da primeira turma avaliada, tendo sido posteriormente concebida, por isso a maior diferença no número de respondentes.

Para viabilizar a análise estatística utilizada, a variável dependente "importância atribuída" foi dicotomizada como "importante" (agregação das respostas "muito importante" e "importante") e "não importante" (agregação das respostas "razoável", "indiferente" e "muito indiferente").

Informações relativas à disponibilidade de materiais, características físicas e funcionamento das unidades sob a perspectiva dos alunos, forneceram as variáveis independentes. Cada uma dessas perguntas foi respondida pelos estudantes como "sim", "não" e "às vezes", sendo esta considerada como "não" para permitir a dicotomização (quadro 1). 
Quadro 1- Síntese das questões relativas às USF.

Os profissionais dispõem de material de biossegurança?

Falta material de consumo?

Os instrumentos estão em boas condições?

A estrutura física é adequada?

A limpeza e higienização são compatíveis com a prestação de serviços de saúde?

A qualidade dos produtos utilizados é compatível com a execução de procedimentos satisfatórios?

Os funcionários respondem às dúvidas e aos questionamentos dos pacientes de modo satisfatório?

Os usuários ficam satisfeitos com o tipo de atenção prestada a eles?

Todos os pacientes que procuram atendimento na USF conseguem ser atendidos na mesma (acesso)?

Existe fila de espera para receber atendimento na USF?

Inicialmente foram utilizados os testes de qui-quadrado e exato de Fisher em nível de significância de 5\% para analisar a associação entre as variáveis independentes e a importância atribuída ao estágio. As variáveis que apresentaram significância estatística de $20 \%$ ou menos na análise bivariada foram testadas na análise de regressão logística múltipla com procedimento stepwise, permanecendo no modelo aquelas com $\mathrm{p} \leq 0,05$. Foram estimados os Odds Ratio (OR) brutos e ajustados e os respectivos intervalos de $95 \%$ de confiança (IC) Todos os testes estatísticos foram realizados com o programa estatístico SAS. (SAS v. 9.2).

$\mathrm{O}$ presente estudo foi submetido e aprovado pelo Comitê de Ética em Pesquisa da Faculdade de Odontologia de Piracicaba sob o protocolo $\mathrm{n}^{\circ}$ 039/2012.

\section{RESULTADOS}

Os estudantes em sua maioria (82\%) atribuíram importância positiva ao estágio para a formação profissional nas turmas avaliadas. Verificou-se a existência de associação entre a falta de importância atribuída ao estágio e a autopercepção sobre disponibilidade de material de biossegurança e de consumo, condições dos instrumentos, adequação de estrutura física, adequação da limpeza e higienização, qualidade dos produtos, resposta dos trabalhadores às dúvidas/questionamentos dos usuários de modo satisfatório, satisfação dos usuários com a atenção prestada a eles, e acesso dos usuários (tabela 1).

A análise de regressão logística múltipla (tabela 2), permite afirmar que a maior probabilidade de considerar o estágio como não importante foi encontrada entre os alunos que apontaram a falta de material de consumo $(\mathrm{OR}=$ 2,17), a limpeza e higienização incompatíveis com a prestação de serviços $(\mathrm{OR}=7,69)$ e àqueles para os quais os funcionários não respondem às dúvidas e questionamentos dos pacientes de modo satisfatório $(\mathrm{OR}=4,34)$.

\section{DISCUSSÃO}

O presente estudo utilizou um instrumento quantitativo de respostas fechadas que, ainda que não permita a livre associação de ideias pelos alunos, sendo limitado por seu caráter mais descritivo e de mensuração, levanta a hipótese de que os alunos que vão para o estágio já concebem a prática em saúde de uma forma mais rigidamente influenciada pelo modo hegemônico de pensamento, dominada pelos 
componentes tecnológicos mais duros, deixando de disponíveis no estágio extramuros, que seriam de compreender os aspectos de singularidade grande valia para fomentar outro tipo de clínica.

Tabela 1. Análise bivariada da associação entre opinião sobre a importância do estágio (dicotomizado entre importante e não importante) e variáveis independentes.

\begin{tabular}{|c|c|c|c|c|c|c|}
\hline \multirow{3}{*}{ Variáveis } & \multicolumn{4}{|c|}{ Estágio extramuros } & \multirow{3}{*}{ OR (IC95\%) } & \multirow{3}{*}{$\mathbf{P}$} \\
\hline & \multicolumn{2}{|c|}{ Importante } & \multicolumn{2}{|c|}{ Não importante } & & \\
\hline & $\mathbf{n}$ & $\%$ & $\mathbf{n}$ & $\%$ & & \\
\hline \multicolumn{7}{|l|}{ Sexo } \\
\hline Feminino & 211 & 80,53 & 51 & 19,47 & & \\
\hline Masculino & 84 & 85,71 & 14 & 14,29 & $0,68(0,36-1,31)$ & 0,3254 \\
\hline \multicolumn{7}{|c|}{ Disponibilidade de materiais de biossegurança } \\
\hline Sim & 263 & 84,29 & 49 & 15,71 & $0,37(0,18-0,75)$ & 0,0096 \\
\hline Não & 28 & 66,67 & 14 & 33,33 & & \\
\hline \multicolumn{7}{|c|}{ Falta de material de consumo } \\
\hline Sim & 86 & 72,88 & 32 & 27,12 & & \\
\hline Não & 203 & 87,12 & 30 & 12,88 & $0,39(0,22-0,69)$ & 0,0016 \\
\hline \multicolumn{7}{|c|}{ Instrumentos em boas condições } \\
\hline Sim & 228 & 85,71 & 38 & 14,29 & $0,43(0,24-0,78)$ & 0,0076 \\
\hline Não & 63 & 72,41 & 24 & 27,59 & & \\
\hline \multicolumn{7}{|c|}{ Estrutura física adequada } \\
\hline Sim & 171 & 87,69 & 24 & 12,31 & $0,36(0,17-0,79)$ & 0,0164 \\
\hline Não & 34 & 72,34 & 13 & 27,66 & & \\
\hline \multicolumn{7}{|c|}{ Limpeza e higienização adequadas } \\
\hline Sim & 285 & 84,07 & 54 & 15,93 & $0,10(0,03-0,32)$ & $<0,0001$ \\
\hline Não & 5 & 35,71 & 9 & 64,29 & & \\
\hline \multicolumn{7}{|c|}{ Produtos de qualidade compatível } \\
\hline Sim & 255 & 85,57 & 43 & 14,43 & $0,29(0,15-0,55)$ & 0,0002 \\
\hline Não & 35 & 63,63 & 20 & 36,36 & & \\
\hline \multicolumn{7}{|c|}{ Funcionários respondem dúvidas dos pacientes } \\
\hline Sim & 282 & 83,93 & 54 & 16,07 & $0,20(0,08-0,49)$ & 0,0004 \\
\hline Não & 12 & 52,17 & 11 & 47,83 & & \\
\hline \multicolumn{7}{|c|}{ Usuários ficam satisfeitos } \\
\hline Sim & 225 & 85,23 & 39 & 14,77 & $0,47(0,27-0,84)$ & 0,0159 \\
\hline Não & 69 & 73,4 & 25 & 26,6 & & \\
\hline \multicolumn{7}{|c|}{ Todos os pacientes têm bom acesso ao serviço } \\
\hline Sim & 185 & 86,05 & 30 & 13,95 & $0,49(0,28-0,84)$ & 0,014 \\
\hline Não & 106 & 75,17 & 35 & 24,83 & & \\
\hline \multicolumn{7}{|c|}{ Fila de espera para atendimento } \\
\hline Sim & 259 & 76,84 & 55 & 23,16 & $0,74(0,32-1,71)$ & 0,6404 \\
\hline Não & 28 & 77,78 & 8 & 22,22 & & \\
\hline Total & 295 & 81,9 & 65 & 18,1 & & \\
\hline
\end{tabular}


Tabela 2. Análise da regressão logística múltipla entre a opinião sobre importância do estágio extramuros para o aluno e as variáveis avaliadas

\begin{tabular}{|c|c|c|c|c|c|c|}
\hline \multirow{3}{*}{ Variáveis } & \multicolumn{4}{|c|}{ Estágio extramuros } & \multirow{3}{*}{ OR ajustado (IC95\%) } & \multirow{3}{*}{$\mathbf{P}$} \\
\hline & \multicolumn{2}{|c|}{ Importante } & \multicolumn{2}{|c|}{ Não importante } & & \\
\hline & n & $\%$ & $\mathbf{n}$ & $\%$ & & \\
\hline \multicolumn{7}{|c|}{ Falta de material de consumo } \\
\hline Sim & 86 & 72,88 & 32 & 27,12 & $2,17(1,20-4,00)$ & 0,0102 \\
\hline Não & 203 & 87,12 & 30 & 12,88 & & \\
\hline \multicolumn{7}{|c|}{ Limpeza e higienização adequadas } \\
\hline Sim & 285 & 84,07 & 54 & 15,93 & & \\
\hline Não & 5 & 35,71 & 9 & 64,29 & $7,69(2,32-25,00)$ & 0,0008 \\
\hline \multicolumn{7}{|c|}{ Funcionários respondem às dúvidas dos pacientes } \\
\hline Sim & 282 & 83,93 & 54 & 16,07 & & \\
\hline Não & 12 & 52,17 & 11 & 47,83 & $4,34(1,63-11,11)$ & 0,0028 \\
\hline
\end{tabular}

*A soma dos respondentes resulta menos de 360 para algumas variáveis devido à perda de dados.

A autopercepção dos alunos sobre uma importância positiva dos estágios extramuros, realizados em diferentes contextos, vem sendo confirmada em distintos estudos, o que vai ao encontro ao verificado, ainda que exista uma grande heterogeneidade de formas de avaliação ${ }^{8,14-18}$. Daher et al. (2012) constataram que alunos participantes de estágio extramuros na atenção básica com enfoque na Odontopediatria, em um contexto com semelhanças àquele vivenciado pelos alunos deste trabalho, atribuíram importância positiva $(92,7 \%)$ num primeiro momento, porém decrescente nos sucessivos retornos ${ }^{19}$.

É importante considerar no presente estudo, porém, a hipótese de os alunos terem emitido uma informação distorcida, gerando resultados mais positivos e otimistas, influenciados pelo julgamento do que seria uma resposta desejável pelos docentes e pósgraduandos do departamento de Odontologia Social, envolvidos na supervisão do estágio, problemática que foi brevemente comentada no estudo de Daher et al. $(2012)^{19}$. Considerar a hipótese de ocorrência desse tipo de viés não minimiza os achados deste e do supracitado estudo, porém demanda que a análise seja feita considerando essa possibilidade. Outra questão a ser ponderada é a pequena proporção de tempo no currículo dedicada ao estágio (64h), pois não é possível garantir que os alunos tenham vivenciado a fundo todas as experiências relacionadas às questões do formulário.

Em relação aos testes de associação, verificou-se que questões ligadas às características de recursos físicos deficitários da unidade estiveram associadas a uma baixa importância atribuída ao estágio pelos alunos. Assim, ao se depararem com um ambiente com recursos materiais diferentes do que esperavam, talvez diferente do ambiente privilegiado encontrado dentro da faculdade, acabaram atribuindo menor importância ao estágio.

A tendência de acadêmicos valorizarem o espaço físico e a disponibilidade de materiais foi relatada em outros contextos. Lynch et al. (2010), em estudo que avaliou as opiniões de graduandos em um centro de ensino extramuros na Inglaterra, realizado em um centro de treinamentos concebido para o ensino que possuía características mais próximas daquelas encontradas nas clínicas pertencentes às 
faculdades, constataram que os alunos valorizaram a disponibilidade de materiais e equipamentos na experiência e criticaram sua falta nos ambientes de ensino intramuros na faculdade $^{8}$. Moimaz et al. (2008) avaliaram a opinião de egressos sobre o Serviço Extramuros Odontológico (SEMO), que presta serviços à população sob os princípios do SUS, constatando que a principal deficiência apontada pelos alunos em relação ao serviço $(63,6 \%)$ foi aquela relacionada à infraestrutura, ou seja, recursos relacionados a equipamentos e materiais de consumo $^{14}$. Sanchez et al. (2008) constataram que a maioria dos alunos de duas faculdades brasileiras atribuiu maior importância ao ensino técnico em estudo de avaliação sobre a percepção de formandos em Odontologia ${ }^{18}$. Tais achados, juntamente aos do presente estudo, corroboram a hipótese de que os alunos costumam valorizar aspectos técnicos das experiências extramurais, sobretudo o uso de alta densidade tecnológica, coerentemente ao ideário odontológico hegemônico.

Segundo Merhy e Franco ${ }^{20}$, o trabalhador de saúde opera um núcleo tecnológico composto pelo Trabalho Morto (instrumental) e o Trabalho Vivo em ato, de forma simultânea, formando certa razão chamada de Composição Técnica do Trabalho. O Trabalho Morto está embutido nos instrumentos, aos quais já se aplicou outrora um trabalho pregresso para sua elaboração, enquanto o Trabalho Vivo é o trabalho feito em ato, campo das tecnologias leves e leves-duras.

Uma boa forma de ilustrar as tecnologias envolvidas no encontro médico-paciente é a noção de que os profissionais (não necessariamente médicos, mas profissionais da saúde em geral como os dentistas) portam valises, pequenas malas, que representam suas caixas de ferramentas tecnológicas: uma vinculada à sua mão, que, no caso do dentista, contém instrumentos como sua seringa carpule, caneta de alta rotação, dentre outros, caracterizada pelas tecnologias duras; outra vinculada à sua cabeça, que contém ferramentas na forma de saberes bem estruturados, como o conhecimento formal sobre um processo infeccioso, denominadas tecnologias levesduras; por fim, uma terceira valise que diz respeito às tecnologias relacionais, que só tem materialidade em ato, na relação terapeutapaciente $^{21}$. Ainda segundo o mesmo autor, não existe configuração única para a disposição de tais caixas de ferramentas, cuja relação difere nos distintos modelos de atenção à saúde. As valises diretamente relacionadas com a singularização do caso clínico, que permitiriam a compreensão do mundo do usuário no projeto terapêutico, seriam as leves-duras, onde localizase o saber estruturado, que pode, desde que não seja engessado pelo modelo de atenção, ser "contaminado" pela singularização do contexto, enquanto um mix de saberes prévios estruturados afetados e modificados pela realidade viva, e as relacionais (leves) ${ }^{21}$. Para a Clínica Ampliada enquanto um dispositivo de humanização na saúde, que procura defender o papel do sujeito e de sua subjetividade, de sua autonomia, a partir de uma prática socialmente mais compreensiva, mais acolhedora, ética, integradora de saberes, faz-se necessário examinar como se relacionam tais valises no processo produtivo em saúde no cenário em questão. Não existem boas chances de realização desta clínica num modelo altamente endurecido, onde os saberes científicos excessivamente estruturados e as tecnologias duras capturem todo o processo, impondo barreiras à compreensão do universo social e das variabilidades ao modus operandi odontológico.

No caso da Odontologia existe uma marcante preponderância das tecnologias duras e leves-duras sobre as leves, cujo currículo é marcado pela ênfase na alta densidade tecnológica, no adestramento manual, na 
valorização da aprendizagem intramuros e laboratorial, organizada segundo subáreas ou especialidades, com pouca ênfase sobre questões sociais e de conceitos de promoção de saúde. Pode-se afirmar que a Composição Técnica do Trabalho hegemônica possui uma razão pendente para o trabalho morto ${ }^{20}$. E, no caso do presente estudo, verificou-se a mesma tendência a partir da frequência das assertivas dos alunos, pois diversas variáveis relacionadas às tecnologias duras (aspectos infraestruturais deficientes) tiveram impacto na percepção dos alunos sobre a importância do estágio, sendo que duas permaneceram associadas na análise de regressão logística (disponibilidade de materiais de consumo e limpeza/higienização).

Evidentemente é desejável viabilizar aos alunos o contato com uma estrutura adequada à prática clínico-cirúrgica em seus cenários de aprendizagem, porém atribuir não-importância ao estágio pelo fato do cenário real do serviço não satisfazer as expectativas em termos estruturais pode sinalizar uma concepção estreita sobre a experiência pedagógica, cuja importância positiva poderia ser, justamente, o desvelamento e melhor compreensão sobre as dificuldades do serviço.

Assim, ressalta-se a necessidade de serem melhores trabalhadas as tecnologias leves no processo de ensino-aprendizagem da Odontologia, almejando uma formação de profissionais de saúde pública capacitados para atender à população, tendo como base também os determinantes sociais da saúde, a humanização e a promoção da saúde.

Além das variáveis relacionadas aos aspectos físicos da unidade de saúde, verifica-se, também, que as variáveis "funcionários não respondem aos questionamentos dos usuários de forma satisfatória", esteve associada a opiniões negativas sobre o estágio. Tal fato pode resultar de uma falta de comprometimento ou empenho por parte da equipe de saúde. Considerando que os exemplos e atitudes são potentes elementos educativos, isso parece também desestimular os alunos. No trabalho de Daher et al. (2012), o não comprometimento da equipe de saúde com os alunos foi associada à uma experiência negativa do estágio ${ }^{19}$. Embora as variáveis sejam diferentes (pois uma diz respeito ao comprometimento da equipe com os alunos e outra com os usuários da unidade), talvez ambas acabem gerando um ambiente desfavorável por expor um desinteresse da equipe ${ }^{18}$. Acreditamos, porém, que exista um potencial de converter a percepção dos alunos no sentido de utilizar essa barreira, comum nos serviços, como substrato para uma reflexão crítica sobre as questões concernentes ao trabalhador da área da saúde como relações as de poder dentro da equipe, desenvolvimento de estresse e burnout.

\section{CONCLUSÕES}

Os resultados obtidos no presente estudo indicam que a maioria dos alunos considerou importante o estágio extramuros nas USF para sua formação profissional. Aqueles que afirmaram que não foi importante parecem levar em consideração aspectos de problemas físicos e materiais nas unidades visitadas, além de perceberem falta de resposta satisfatória da equipe às dúvidas dos usuários.

Uma percepção negativa relacionada aos aspectos do lugar, ainda que estes sejam componentes inerentes do cenário real dos serviços e parte da experiência pedagógica, parece influenciar negativamente a valoração da importância da atividade de estágio. A opinião dos alunos sobre o estágio, influenciada tanto por aspectos experimentados quanto por representações sociais acerca do setor público de saúde que trazem a priori, talvez possa ter um efeito na importância que eles atribuem à experiência. 
A partir do apresentado, sugere-se aprofundar a problematização com os alunos, antes, durante e após o estágio, sobre a importância dos aspectos referentes às tecnologias leves implicadas no processo de trabalho dos profissionais da saúde na atenção básica, ou seja, a comunicação, vínculo, acolhimento, autonomização, dentre outras, fundamentais para o exercício de uma Clínica Ampliada. Provavelmente os alunos trazem para o estágio uma expectativa adquirida no ambiente acadêmico coerente com o modelo privado de prática, sendo função das disciplinas que se propõem a ampliar a visão do aluno prepará-lo para compreender as distintas propostas.

\section{ABSTRACT \\ The valuation of the Supervised Internship in the Family Health Unit by the Dental students: what factors influence their perception?}

The extramural stage is an essential pedagogical strategy used to train the health professions to foster a more comprehensive and socially responsible practice, especially when developed in real public service scenarios. These experiences are not always well accepted by students, so exploring the factors that interfere with this assessment may be useful to improve this activity. The objective of the present study was to explore which students' perceptions influence the assessment regarding the importance of the internship in Family Health Units in their professional training. A total of 185 students who completed their internship during their final years participated in the research. Data were obtained through electronic forms composed of questions about the functioning and working conditions in the units (independent variables) and by the importance attributed to the stage (dependent variable). The students who perceived lack of consumption material $(\mathrm{OR}=$ $2.17, \mathrm{CI}=1.20-4.00$ ), cleaning and sanitation were incompatible with the provision of health services $(\mathrm{OR}=7.69,=2.32-25.00)$ and the lack of satisfactory answers from the health team staff to patients $(\mathrm{OR}=4.34, \mathrm{CI}=1.63-11.11)$ were more likely to consider the experience as nonimportant. We concluded that infrastructural problems and aporias of daily services seem to influence in a negative evaluation of the stage by the students, pointing out the need for a more significant support of the faculty to turn this reading of reality into important pedagogical products to the students.

Descriptors: Education, Dental. Staff Development. Unified Health System. Family Health.

\section{REFERÊNCIAS}

1. Marques RM, Mendes Á. Atenção Básica e Programa de Saúde da Família (PSF): novos rumos para a política de saúde e seu financiamento? Ciênc Saúde Colet. 2003;8(2):403-15.

2. Mialhe FL, Meneghim MDC, Souza M da LR, Pereira AC. Integração ensino-serviço para a reorientação da formação profissional em saúde: a experiência da FOP/Unicamp. Rio de Janeiro: Usina de Letras; 2011.

3. Villalba JP, Madureira PR De. Perfil profissional do cirurgião-dentista para atuação no Sistema Único de Saúde (SUS). Rev Inst Ciênc Saúde. 2009;27 (3):262-8.

4. Brasil. Programa Nacional de Reorientação da Formação Profissional em Saúde - Pró-Saúde: objetivos, implementação e desenvolvimento potencial/Ministério da Saúde, Ministério da Educação. Brasília: Ministério da Saúde, 2005. 86p.

5. Zilbovicius C, De Araujo ME, Botazzo C, Frias AC, Junqueira SR, Junqueira CR. A paradigm shift in predoctoral dental curricula in Brazil: evaluating the process of change. J Dent Educ. 2011;75(4):55764.

6. Schönwetter DJ, Law D, Mazurat R, 
Sileikyte R, Nazarko O. Assessing graduating dental students' competencies: the impact of classroom, clinic and externships learning experiences. Eur $\mathbf{J}$ Dent Educ. 2011;15(3):142-52.

7. Mascarenhas AK. Community-based Dental education at Boston University. J Dent Educ. 2011;75(10,S):S21-S24.

8. Lynch CD, Ash PJ, Chadwick BL. Student perspectives and opinions on their experience at an undergraduate outreach dental teaching centre at Cardiff: a 5-year study. Eur J Dent Educ. 2010;14(1):12-6.

9. Piskorowski WA, Fitzgerald M, Mastey J, Krell RE. Development of a sustainable community-based Dental Education Program. J Dent Educ. 2011;75(8):103843.

10. Campos GWS. Reforma política e sanitária: a sustentabilidade do sus em questão? Ciênc Saúde Colet. 2007;12(2) :301-6.

11. Batista MJ, Gibilini C, Kobayashi HM, Ferreira LL, Silva C, Rosário L. Relato de experiência da interação entre universidade, comunidade e unidade de saúde da família em Piracicaba, SP, Brasil. Arq Odontol. 2010;46:144-51.

12. Leme PAT, Pereira AC, Meneghim MC, Mialhe FL. Perspectivas de graduandos em odontologia acerca das experiências na atenção básica para sua formação em saúde. Ciênc Saúde Colet. 2015;20(4) :1255-65.

13. Meneghim MC, Pereira AC, Mialhe FL, Sousa MLR. Pró-Saúde e os quarenta anos de integração docente-assistencial da Faculdade de Odontologia de Piracicaba Unicamp. Rev Bras Educ Méd. 2012;36(1):97-104.

14. Moimaz SAS, Saliba NA, Garbin CAS, Zina LG. Atividades extramuros na ótica de egressos do curso de graduação em odontologia. Rev ABENO. 2008;8:23-9.

15. Moimaz SAS, Saliba NA, Garbin CAS, Zina LG, Furtado JF, Amorin JA de. Serviço extramuro odontológico: impacto na formação profissional. Pesqui Bras. Odontopediatria Clín Integr. 2004;4(1) :53-7.

16. Cavalcanti YW, Cartaxo RDO, Wilney W, Padilha N. Educação odontológica e Sistema de Saúde brasileiro: práticas e percepções de estudantes de graduação. Arq Odontol. 2010;46:224-31.

17. Maguire A, Hind V, Waterhouse PJ, Tabari D, Steen IN, Lloyd J. Developing a primary dental care outreach (PDCO) course - part 2: perceptions of dental students. Eur J Dent Educ. 2009;(4):210-7.

18. Sanchez HF, Drumond MM, Vilaça ÊL. Adequação de recursos humanos ao PSF: percepção de formandos de dois modelos de formação acadêmica em Odontologia. Ciênc Saúde Colet. 2008;13(2):523-31.

19. Daher A, Costa LR, Machado GCM. Dental Students' perceptions of community-based education: a retrospective study at a Dental School in Brazil. J Dent Educ. 2012;76(9):1218-25.

20. Merhy EE, Franco TB. Cartografias do trabalho e cuidado em saúde. Tempus. 2012;6(2):151-63

21. Merhy EE. Um ensaio sobre o médico e suas valises tecnológicas. Contribuições para compreender as reestruturações produtivas do setor Saúde. Interface Comum Saúde Educ. 2000;4(6):109-16.

Correspondência para:

Pedro Augusto Thiene Leme email: pedroleme3@gmail.com Rua Vital Brasil, 150 - Caixa Postal 1170

Cidade Universitária Zeferino Vaz

Barão Geraldo 13083-888 Campinas/SP 